\title{
Chronic Lymphocytic Leukaemia
}

\author{
Olivier Tournilhac and Peter Dreger
}

\section{Clinical Development of CAR-T Cells for CLL}

Although chronic lymphocytic leukaemia (CLL) was one of the first two entities in which CAR-T cells were evaluated, it has not yet arrived in the clinical routine. Since the landmark study by Porter et al. (2011), only six CLL-specific clinical trials have been published, altogether comprising no more than 155 patients (Porter et al. 2015; Gill et al. 2018; Turtle et al. 2017; Gauthier et al. 2020; Siddiqi et al. 2020; Wierda et al. 2020; Frey et al. 2020). All six of these studies investigated CD19-directed CAR-T constructs in heavily pretreated patients, mostly having failed BTKi +/- venetoclax therapy. Despite overall response rates of 60-95\%, including MRD clearance in a large proportion of patients, the CR rates appear to be relatively low, and only a few durable responses have been reported in patients achieving a CR (Porter et al. 2015; Frey et al. 2020; Cappell et al. 2020). While toxicity includes 5-20\% grade 3 cytokine release syndrome and 5-25\% grade 3 neurotoxicity and appears manageable, long-term efficacy remains an unresolved issue. CLL-specific efficacy barriers for CD19 CAR-T cells could include a reduced capacity for sustained $\mathrm{T}$ cell expansion in extensively pretreated elderly CLL patients (Lemal and Tournilhac 2019), along with impaired T cell motility, impaired T cell mitochondrial fitness, and T cell exhaustion (Bair and Porter 2019). Concurrent use of ibrutinib might reduce the CRS rate and severity (Gauthier et al. 2020; Gill et al. 2018; Wierda et al. 2020) without impairing CAR-T cell expansion.

\footnotetext{
O. Tournilhac $(\bowtie)$

Service d'Hematologie Clinique et de Therapie Cellulaire, CHU, Universite Clermont Auvergne, Clermont Ferrand, France

e-mail: otournilhac@ @ chu-clermontferrand.fr

P. Dreger

Department of Medicine V, Hematology, Oncology and Rheumatology, University Hospital Heidelberg, Heidelberg, Germany

e-mail: peter.dreger@med.uni-heidelberg.de
} 


\section{Current Indications for CAR-T Cells in the Treatment Landscape of CLL}

In the absence of studies with informative sample sizes and follow-up and without an approved CAR-T cell preparation available, there is currently no indication for CAR-T cells in CLL outside of a clinical trial. However, if a suitable trial is available, CAR-T cells can be proposed as an alternative in patients with high-risk-2 CLL who have a high transplant risk according to the EBMT-ERIC recommendations (Dreger et al. 2018). In patients with a low transplant risk, allogeneic haematopoietic cell transplantation (alloHCT) still appears to be the more promising approach in terms of long-term disease control (Tournilhac et al. 2020; Roeker et al. 2020; Mato et al. 2020). The advent of more effective CAR-T cell therapies for CLL is eagerly awaited and may rapidly change this algorithm.

- Currently, there is no standard indication for CAR-T cells in CLL.

- CAR-T cells may be an alternative to alloHCT in high-risk patients in clinical trials.

\section{Prospective Studies of Autologous Anti-CD19 CAR-T Cell Therapy for CLL}

\begin{tabular}{l|l|l|l|l|l|l|l}
\hline & $\begin{array}{l}\text { Porter } \\
\text { et al. } \\
(2015)\end{array}$ & $\begin{array}{l}\text { Frey } \\
\text { et al. } \\
(2020)\end{array}$ & $\begin{array}{l}\text { Gill } \\
\text { et al. } \\
(2018)\end{array}$ & $\begin{array}{l}\text { Turtle } \\
\text { et al. } \\
(2017)\end{array}$ & $\begin{array}{l}\text { Gauthier } \\
\text { et al. } \\
(2020)\end{array}$ & $\begin{array}{l}\text { Siddiqi } \\
\text { et al. } \\
(2020)\end{array}$ & $\begin{array}{l}\text { Wierda } \\
\text { et al. } \\
(2020)\end{array}$ \\
\hline Patients $(n)$ & 14 & 38 & 19 & $24(5 \mathrm{RT})$ & $19(4 \mathrm{RT})$ & $22(1 \mathrm{RT})$ & 19 \\
\hline $\begin{array}{l}\text { CAR-T } \\
\text { with } \\
\text { ibrutinib }\end{array}$ & $\begin{array}{l}\text { CTL019 } \\
\text { No }\end{array}$ & $\begin{array}{l}\text { CART- } \\
19 \\
\text { No }\end{array}$ & $\begin{array}{l}\text { CTL119 } \\
\text { Yes }\end{array}$ & $\begin{array}{l}\text { JCAR014 } \\
\text { No }\end{array}$ & $\begin{array}{l}\text { JCAR014 } \\
\text { Yes }\end{array}$ & $\begin{array}{l}\text { JCAR017 } \\
\text { No }\end{array}$ & $\begin{array}{l}\text { JCAR017 } \\
\text { Yes }\end{array}$ \\
\hline Age (years $)$ & $66(51-78)$ & $\begin{array}{l}61 \\
(49- \\
76)\end{array}$ & $\begin{array}{l}62 \\
(42-76)\end{array}$ & $\begin{array}{l}61 \\
(40-73)\end{array}$ & $\begin{array}{l}65 \\
(40-71)\end{array}$ & $66(50-80)$ & $\begin{array}{l}60 \\
(50-77)\end{array}$ \\
\hline $\begin{array}{l}\text { Previous } \\
\text { lines (n) }\end{array}$ & $5(1-11)$ & $\begin{array}{l}3.5 \\
(2-7)\end{array}$ & $2(1-16)$ & $5(3-9)$ & $5(1-10)$ & $4(2-11)$ & $4(2-11)$ \\
\hline $\begin{array}{l}\text { Ibrutinib } \\
(\mathrm{R} / \mathrm{R})\end{array}$ & $1(1)$ & $9(?)$ & $5(0)$ & $24(19)$ & $19(19)$ & $23(17)$ & $19(19)$ \\
\hline $\begin{array}{l}\text { Venetoclax } \\
(\mathrm{R} / \mathrm{R})\end{array}$ & 0 & 1 & 0 & $6(6)$ & $11(6)$ & $13(11)$ & $11(\mathrm{na})$ \\
\hline CK $(\%)$ & $\mathrm{Na}$ & Na & Na & 67 & 74 & 48 & 42 \\
\hline $\begin{array}{l}\text { TP53 alt. } \\
(\%)\end{array}$ & 43 & $39^{\mathrm{b}}$ & 58 & Del $=58$ & Del $=74$ & $\begin{array}{l}\text { Mut }=61 \\
\text { Del }=35\end{array}$ & $\begin{array}{l}\text { Mut }=32 \\
\text { Del }=42\end{array}$ \\
\hline ORR (\%) & 57 & $44^{\mathrm{b}}$ & $71^{\mathrm{b}}$ & 70 & $83^{\mathrm{b}}$ & $82^{\mathrm{b}}$ & 95 \\
\hline CR $(\%)$ & 29 & $28^{\mathrm{b}}$ & $43^{\mathrm{b}}$ & 17 & $22^{\mathrm{b}}$ & $46^{\mathrm{b}}$ & 63 \\
\hline $\begin{array}{l}\text { MRD(-) } \\
\text { BM (\%) }\end{array}$ & 29 & na & $78^{\mathrm{b}}$ & $50^{\mathrm{b}}$ & $61^{\mathrm{b}}$ & $65^{\mathrm{b}}$ & 79 \\
\hline
\end{tabular}




\begin{tabular}{|c|c|c|c|c|c|c|c|}
\hline & $\begin{array}{l}\text { Porter } \\
\text { et al. } \\
\text { (2015) }\end{array}$ & $\begin{array}{l}\text { Frey } \\
\text { et al. } \\
\text { (2020) }\end{array}$ & $\begin{array}{l}\text { Gill } \\
\text { et al. } \\
\text { (2018) }\end{array}$ & $\begin{array}{l}\text { Turtle } \\
\text { et al. } \\
\text { (2017) }\end{array}$ & $\begin{array}{l}\text { Gauthier } \\
\text { et al. } \\
(2020)\end{array}$ & $\begin{array}{l}\text { Siddiqi } \\
\text { et al. } \\
(2020)\end{array}$ & $\begin{array}{l}\text { Wierda } \\
\text { et al. } \\
\text { (2020) }\end{array}$ \\
\hline $\begin{array}{l}\text { CRS (all/ } \\
\text { G3) (\%) }\end{array}$ & $64 / 43$ & $63 / 24$ & $95 / 16$ & $83 / 8$ & $74 / 0$ & $74 / 9$ & $74 / 5$ \\
\hline $\begin{array}{l}\text { NT (all/ } \\
\text { G3) (\%) }\end{array}$ & $36 / 7$ & na/8 & $26 / 5$ & $33 / 25$ & $26 / 26$ & $39 / 22$ & $32 / 16$ \\
\hline $\mathrm{FU}(\mathrm{m})$ & $19(6-53)$ & $\begin{array}{l}32 \\
(2-75)\end{array}$ & $\begin{array}{l}19 \\
(8-28)\end{array}$ & NA & $12(4-17)$ & 24 & 10 \\
\hline $\begin{array}{l}\text { PFS (m) } \\
\text { PFS }>24 m \\
\text { (n) }\end{array}$ & $\begin{array}{l}28 \% \\
@ 18 \mathrm{~m} \\
3\end{array}$ & $\begin{array}{l}1 \mathrm{~m} \\
7\end{array}$ & $\begin{array}{l}\text { na } \\
\text { na }\end{array}$ & $\begin{array}{l}8.5 \mathrm{~m} \\
\text { na }\end{array}$ & $\begin{array}{l}38 \% \\
@ 12 \mathrm{~m} \\
\text { na }\end{array}$ & $\begin{array}{l}50 \% \\
@ 18 \mathrm{~m} \\
\text { na }\end{array}$ & $\begin{array}{l}\text { na } \\
\text { na }\end{array}$ \\
\hline $\begin{array}{l}\text { NRM (n) } \\
\text { cause }\end{array}$ & 1 & $\begin{array}{l}0 \\
\text { / }\end{array}$ & $\begin{array}{l}1 \\
\text { (cardiac) }\end{array}$ & $\begin{array}{l}1 \\
\text { (CRS/ } \\
\text { NT) }\end{array}$ & $\begin{array}{l}1 \\
\text { (cardiac) }\end{array}$ & $\begin{array}{l}0 \\
/\end{array}$ & $\begin{array}{l}0 \\
\text { / }\end{array}$ \\
\hline
\end{tabular}

$R T$ Richter transformation, $R / R$ relapsed/refractory, TP53 alt. TP53 mutation and/or 17p deletion, $C R S$ cytokine release syndrome, $N T$ neurotoxicity, all $G$ all grades, $G \geq 3$ grade $\geq 3$, na not available, $C K$ complex karyotype ( $\geq 3$ abnormalities), $B M$ bone marrow, $M R D(-) B M$ negative bone marrow minimal residual disease, $N R M$ non relapse mortality

${ }^{a}$ Transcend CLL 004 study with lisocabtagene maraleucel

${ }^{\mathrm{b}}$ Assessment limited to evaluable patients

\section{Key Points}

- Autologous CAR-T cells for CLL have been in development for almost 10 years, with interesting results in poor-risk disease, including patients double refractory to both BTKi and BCL2i.

- However, more data, including clinical trials with a longer follow-up time, are required before adding CAR-T cells to clinical practice.

\section{References}

Bair SM, Porter DL. Accelerating chimeric antigen receptor therapy in chronic lymphocytic leukemia: the development and challenges of chimeric antigen receptor T-cell therapy for chronic lymphocytic leukemia. Am J Hematol. 2019;94(Suppl_1):S10-7.

Cappell KM, Sherry RM, Yang JC, et al. Long-term follow-up of anti-CD19 chimeric antigen receptor T-cell therapy. JCO. 2020;38(32):3805-15.

Dreger P, Ghia P, Schetelig J, et al. High-risk chronic lymphocytic leukemia in the era of pathway inhibitors: integrating molecular and cellular therapies. Blood. 2018;132(9):892-902.

Frey NV, Gill S, Hexner EO, et al. Long-term outcomes from a randomized dose optimization study of chimeric antigen receptor modified $\mathrm{T}$ cells in relapsed chronic lymphocytic leukemia. JCO. 2020;38:2862.

Gauthier J, Hirayama AV, Purushe J, et al. Feasibility and efficacy of CD19-targeted CAR-T cells with concurrent ibrutinib for CLL after ibrutinib failure. Blood. 2020;135(19):1650-60.

Gill SI, Vides V, Frey NV, et al. Prospective clinical trial of anti-CD19 CAR-T cells in combination with Ibrutinib for the treatment of chronic lymphocytic leukemia shows a high response rate. Blood. 2018;132(Suppl 1):298. 
Lemal R, Tournilhac O. State-of-the-art for CAR-T cell therapy for chronic lymphocytic leukemia in 2019. J Immunother Cancer. 2019;7(1):202.

Mato AR, Roeker LE, Jacobs R, et al. Assessment of the efficacy of therapies following Venetoclax discontinuation in CLL reveals BTK inhibition as an effective strategy. Clin Cancer Res. 2020;26(14):3589-96.

Porter DL, Levine BL, Kalos M, Bagg A, June CH. Chimeric antigen receptor-modified T cells in chronic lymphoid leukemia. N Engl J Med. 2011;365(8):725-33.

Porter DL, Hwang W-T, Frey NV, et al. Chimeric antigen receptor T cells persist and induce sustained remissions in relapsed refractory chronic lymphocytic leukemia. Sci Transl Med. 2015;7(303):303ra139.

Roeker LE, Dreger P, Brown JR, et al. Allogeneic stem cell transplantation for chronic lymphocytic leukemia in the era of novel agents. Blood Adv. 2020;4(16):3977-89.

Siddiqi T, Soumerai JD, Dorritie KA, et al. Updated follow-up of patients with relapsed/refractory chronic lymphocytic leukemia/small lymphocytic lymphoma treated with Lisocabtagene Maraleucel in the phase 1 monotherapy cohort of transcend CLL 004, including high-risk and Ibrutinib-treated patients. Blood 2020;136(1):546.

Tournilhac O, Le Garff-Tavernier M, Nguyen Quoc S, et al. Efficacy of minimal residual disease driven immune-intervention after allogeneic hematopoietic stem cell transplantation for highrisk chronic lymphocytic leukemia: results of a prospective multicentric trial. Haematologica. 2020; https://doi.org/10.3324/haematol.2019.239566.

Turtle CJ, Hay KA, Hanafi L-A, et al. Durable molecular remissions in chronic lymphocytic leukemia treated with CD19-specific chimeric antigen receptor-modified $\mathrm{T}$ cells after failure of Ibrutinib. J Clin Oncol. 2017;35(26):3010-20.

Wierda W, Dorritie KA, Munoz J, et al. Transcend CLL 004: phase 1 Cohort of Lisocabtagene Maraleucel (liso-cel) in combination with Ibrutinib for patients with Relapsed/Refractory (R/R) chronic lymphocytic leukemia/small lymphocytic lymphoma (CLL/SLL). Blood 2020;136(1):544.

Open Access This chapter is licensed under the terms of the Creative Commons Attribution 4.0 International License (http://creativecommons.org/licenses/by/4.0/), which permits use, sharing, adaptation, distribution and reproduction in any medium or format, as long as you give appropriate credit to the original author(s) and the source, provide a link to the Creative Commons license and indicate if changes were made.

The images or other third party material in this chapter are included in the chapter's Creative Commons license, unless indicated otherwise in a credit line to the material. If material is not included in the chapter's Creative Commons license and your intended use is not permitted by statutory regulation or exceeds the permitted use, you will need to obtain permission directly from the copyright holder.

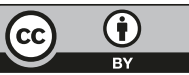

